\title{
Fast Patch-Based Pseudo-CT Synthesis from T1-Weighted MR Images for PET/MR Attenuation Correction in Brain Studies
}

\author{
Angel Torrado-Carvajal ${ }^{1,2}$, Joaquin L. Herraiz ${ }^{2,3}$, Eduardo Alcain ${ }^{4}$, Antonio S. Montemayor ${ }^{4}$, Lina Garcia-Cañamaque ${ }^{5}$, \\ Juan A. Hernandez-Tamames ${ }^{1,2}$, Yves Rozenholc ${ }^{6}$, and Norberto Malpica ${ }^{1,2}$ \\ ${ }^{I}$ Medical Image Analysis and Biometry Laboratory, Universidad Rey Juan Carlos, Móstoles, Madrid, Spain; ${ }^{2}$ Madrid-MIT M+Visión \\ Consortium, Madrid, Spain; ${ }^{3}$ Research Laboratory of Electronics, Massachusetts Institute of Technology, Cambridge, Massachusetts; \\ ${ }^{4}$ Department of Computer Science, Universidad Rey Juan Carlos, Móstoles, Madrid, Spain, ${ }^{5}$ Hospital Universitario HM Puerta del \\ Sur, HM Hospitales, Móstoles, Madrid, Spain; and ${ }^{6}$ MAP5, CNRS UMR 8145, University Paris Descartes, Paris, France
}

Attenuation correction in hybrid PET/MR scanners is still a challenging task. This paper describes a methodology for synthesizing a pseudoCT volume from a single T1-weighted volume, thus allowing us to create accurate attenuation correction maps. Methods: We propose a fast pseudo-CT volume generation from a patient-specific MR T1weighted image using a groupwise patch-based approach and an MRI-CT atlas dictionary. For every voxel in the input MR image, we compute the similarity of the patch containing that voxel to the patches of all MR images in the database that lie in a certain anatomic neighborhood. The pseudo-CT volume is obtained as a local weighted linear combination of the CT values of the corresponding patches. The algorithm was implemented in a graphical processing unit (GPU). Results: We evaluated our method both qualitatively and quantitatively for PET/MR correction. The approach performed successfully in all cases considered. We compared the SUVs of the PET image obtained after attenuation correction using the patient-specific CT volume and using the corresponding computed pseudo-CT volume. The patient-specific correlation between SUV obtained with both methods was high $\left(R^{2}=0.9980, P<0.0001\right)$, and the Bland-Altman test showed that the average of the differences was low $(0.0006 \pm$ $0.0594)$. A region-of-interest analysis was also performed. The correlation between $S U V_{\text {mean }}$ and $S U V_{\text {max }}$ for every region was high $\left(R^{2}=\right.$ 0.9989, $P<0.0001$, and $R^{2}=0.9904, P<0.0001$, respectively). Conclusion: The results indicate that our method can accurately approximate the patient-specific CT volume and serves as a potential solution for accurate attenuation correction in hybrid PET/MR systems. The quality of the corrected PET scan using our pseudo-CT volume is comparable to having acquired a patient-specific CT scan, thus improving the results obtained with the ultrashort-echo-timebased attenuation correction maps currently used in the scanner. The GPU implementation substantially decreases computational time, making the approach suitable for real applications.

Key Words: pseudo-CT; image synthesis; PET/MR; attenuation correction; GPU

J Nucl Med 2016; 57:136-143

DOI: $10.2967 /$ jnumed.115.156299

Received Feb. 20, 2015; revision accepted Oct. 7, 2015.

For correspondence contact: Angel Torrado-Carvajal, Medical Image Analysis and Biometry Laboratory, Universidad Rey Juan Carlos, C/Tulipán s/n, 28933, Móstoles, Madrid, Spain.

E-mail: angel.torrado@urjc.es

Published online Oct. 22, 2015.

COPYRIGHT $\odot 2016$ by the Society of Nuclear Medicine and Molecular Imaging, Inc.
$\mathbf{P}$ hilation $\gamma$-rays emitted indirectly by a positron-emitting radionuclide (radiotracer), allowing measurement of its biodistribution. MRI provides, among others, detailed morphologic imaging of organs or soft tissue, with excellent spatial resolution. The evolution of both modalities into complementary in vivo molecular imaging techniques has generated increased interest in the development of hybrid MR/PET systems (1).

The construction of accurate attenuation correction (AC) maps is essential for the clinical application of hybrid PET/MR systems. Constructing PET AC maps for use in these hybrid systems is challenging because no direct relation exists between PET attenuation coefficients $(\mu)$ and MR signal intensity (2), contrary to what happens with AC maps and the intensity of CT images (3). Additionally, treating bone as soft tissue in MR-derived AC maps for PET/MR AC leads to a substantial underestimation in the analysis of PET tracer distribution (4). Creating a pseudo-CT volume from MRI data could help to produce an accurate AC map comparable in quality to one acquired from a real CT scan of the patient.

Previous approaches to MRI-based AC have been presented in the literature (5). They differ mainly in the type of semantic representation used to describe the image data, based on mathematic morphology (6), deformable models (7), MRI Dixon (8) or ultrashort-echo-time (TE) sequences $(9,10)$, and multiatlas segmentation using label fusion (11). However, most of these methods showed limited accuracy when used to create AC maps (12). Hofmann et al. combined local pattern recognition with image registration to generate pseudo-CT images (13). Recently, Izquierdo-Garcia et al. proposed an approach to pseudo-CT synthesis based on nonrigid registration to an atlas using the standard SPM8 software with a CT template (14). Burgos et al. described an improved method that uses nonrigid registration to an atlas, followed by label fusion based on patch-similarity measurements (15). Furthermore, clinical scanners currently rely on acquisition of multiple MR sequences, but the results are not fully reliable (16).

Patch-based methods have proven to be a versatile segmentation technique (17). Patch-based segmentation was introduced as an alternative to label propagation that eliminates the requirement for nonrigid registration. The technique is an adaptation of the nonlocal framework developments for nonlocal denoising (18). Several approaches have been introduced depending on the patch fusion methods or rules $(19,20)$. Ye et al. proposed a patch-based method for generating a T2-weighted volume from a T1-weighted volume (21). 
In this work, we propose a simple and fast approach to pseudo-CT synthesis from a single patient-specific MR T1-weighted image using a groupwise patch-based approach and a limited MRI and CT atlas dictionary. Our method is purely patch-based, eliminating the need for a registration step and considerably reducing time. Also, because the algorithm becomes parallelizable, a fast implementation using a graphical processing unit (GPU) card is presented.

In the next section we describe the dataset used in this work and the overall process of the pseudo-CT synthesis and AC maps. Then, we present different experiments quantitatively comparing the use of patient-specific CT and our pseudo-CT volumes for AC correction. Finally we discuss the findings from the experimental results and the possible implications for managing PET AC in hybrid PET/MR systems, and we draw some conclusions.

\section{MATERIALS AND METHODS}

\section{Patient Population}

The institutional review board-approved retrospective study included a dataset containing 19 healthy women (mean age, $34.96 \pm 5.23 \mathrm{y}$; range, 27-46 y). All underwent both MR and CT imaging. For this type of study, the need for written informed consent was waived.

\section{Image Acquisition}

MR images of the head were acquired on a Signa HDxt 3.0-T MR scanner (GE Healthcare) using the body coil for excitation and an 8-channel quadrature brain coil for reception. Imaging was performed using an isotropic 3-dimensional (3D) T1-weighted spoiled gradient recalled sequence with a repetition time of $10.024 \mathrm{~ms}$, TE of $4.56 \mathrm{~ms}$, inversion time of $600 \mathrm{~ms}, 1$ excitation, acquisition matrix of $288 \times 288$, resolution of $1 \times 1 \times 1 \mathrm{~mm}$, and flip angle of $12^{\circ}$.

Low-dose CT images were acquired on a Somatom Sensation 16 CT scanner (Siemens) with a matrix of $512 \times 512$, resolution of $0.48 \times$ $0.48 \mathrm{~mm}$, slice thickness of $0.75 \mathrm{~mm}$, pitch of $0.7 \mathrm{~mm}$, acquisition angle of $0^{\circ}$, voltage of $120 \mathrm{kV}$, and radiation intensity of $200 \mathrm{~mA}$.

\section{Data Preprocessing}

Images were preprocessed using 3D Slicer built-in modules (22). This preprocessing included MRI bias correction (N4 bias correction tool in the Insight Toolkit), rigid registration (general registration BRAINS) to align each patient-specific MRI-CT pair, rigid registration to prealign all the images in the same spatial origin-all the images are set in the same orientation - and normalization of the grayscale values (Insight Toolkit-based histogram matching).

\section{Pseudo-CT Synthesis}

Weights Estimation. Let $I$ be an input image to be processed, and $A$ an anatomy atlas containing a set of MRI T1-weighted volumes $I_{\mathrm{MR}}$ and their corresponding $I_{\mathrm{CT}}$ volumes: $A=\left\{\left(I_{\mathrm{MR}}^{i}, I_{\mathrm{CT}}^{i}\right) i=1, \ldots, n\right\}$.

Let us consider $w_{i}$ as a weight reflecting nonlocal similarities between voxels $\boldsymbol{a}=\left(a_{x}, a_{y}, a_{z}\right)$ in the input image $I$ and voxels $\boldsymbol{b}=\left(b_{x}, b_{y}, b_{z}\right)$ in the image $I_{\mathrm{MR}}^{i}$ of the atlas $A$ over the image domain $\boldsymbol{\Omega}$, and computed using the following equation:

$$
w_{i}(\boldsymbol{a}, \boldsymbol{b})=f\left(\frac{\sum_{\boldsymbol{a}^{\prime} \in P_{I}(\boldsymbol{a}), \boldsymbol{b}^{\prime} \in P_{I_{\mathrm{MR}}^{i}}(\boldsymbol{b})}\left(I\left(\boldsymbol{a}^{\prime}\right)-I_{\mathrm{MR}}^{i}\left(\boldsymbol{b}^{\prime}\right)\right)^{2}}{2 S \beta \hat{\sigma}^{2}}\right)
$$

where $P_{I}(\boldsymbol{a})$ is a 3D patch of the image $I$ centered at voxel $\boldsymbol{a}, S$ is the number of voxels in the 3D patch, $\beta$ is a smoothing parameter, and $\hat{\sigma}$ is the SD of the noise. The original denoising approach assumes that similarities of a patch can be found over the entire image domain $\boldsymbol{\Omega}$. However, in our context of modality propagation, the variations of the anatomic structures in a population are bounded, and we can thus find good matches in a specific neighborhood $N_{m \times m \times m}(\boldsymbol{a})$ of a certain voxel $\boldsymbol{a}$. The weights are then estimated in this local area as $w=\{w(\boldsymbol{a}, \boldsymbol{b})$, $\left.\forall \boldsymbol{a} \in \Omega, \boldsymbol{b} \in N_{m \times m \times m}(\boldsymbol{a})\right\}$, reflecting the local similarities between $I$ and $\left\{I_{\mathrm{MR}}^{i}\right\}_{i=1, \ldots, n}$.

The size of the patch has been set to $3 \times 3 \times 3$ for all experiments. The size of the considered neighborhood has been set to $11 \times 11 \times 11$, which is directly related to the anatomic variability of the head (21).

Groupwise Label Propagation. Once the weights have been computed using Equation 1, they can be used to estimate the corresponding voxel $\boldsymbol{a}$ of image $I_{\text {pseudo-CT }}$ as a weighted linear groupwise combination of the patches in $I_{\mathrm{CT}}^{i}$ as follows:

$$
\forall \boldsymbol{a} \in \Omega, I_{\text {pseudo-CT }}(\boldsymbol{a})=\frac{\sum_{i=1}^{n} \sum_{\boldsymbol{b} \in N_{m \times m \times m}(\boldsymbol{a})} w(\boldsymbol{a}, \boldsymbol{b}) I_{C T}^{i}(\boldsymbol{b})}{\sum_{i=1}^{n} \sum_{\boldsymbol{b} \in N_{m \times m \times m}}(\boldsymbol{a})},
$$

which takes all the images $I_{\mathrm{MR}}^{i}$ in the atlas $A$ to produce a groupwise combination of $I_{\mathrm{CT}}^{i}$, thus obtaining an estimation of $I_{\text {pseudo-CT }}$.

Regularization. Contrary to registration-based approaches, if there is no correspondence between the patch of the input image $I$ and the patches of the images $I_{\mathrm{MR}}^{i}$ in the atlas $A$, no value (NaN due to division by 0 ) is assigned to the voxel $\boldsymbol{a}$ of $I_{\text {pseudo-CT. This situation makes it necessary to }}$ include a regularization step that deals with nonlabeled voxels. Because these cases are usually isolated, in this work we have assigned to this voxel the value of the median in its neighborhood. The size of this new neighborhood has been set to 3 in this paper: $N_{3 \times 3 \times 3}(\boldsymbol{a})$.

$$
\forall \boldsymbol{a}=\boldsymbol{N a N} \in \Omega, I_{\text {pseudo-CT }}(\boldsymbol{a})=\operatorname{median}_{N_{3 \times 3 \times 3}(\boldsymbol{x})}\left(I_{\mathrm{pseudo}-\mathrm{CT}}(\boldsymbol{a})\right) . \quad \text { Eq. } 3
$$

\section{GPU Implementation}

The patch-based algorithm is highly parallelizable. The calculation of the resulting $I_{\text {pseudo-CT }}$ for each voxel $I$ in the $3 \mathrm{D}$ input volume is completely independent from that for the other voxels. Configuring a 3D grid in a CUDA (Compute Unified Device Architecture) model allows loops to be eliminated in order to iterate over the different voxels in the volume. Our CUDA kernel contains the selection of atlas A and the search in the neighborhood $N$; in this way, each label calculation is performed in a separate thread.

\section{PET Simulation}

We obtained detailed tissue-segmented models from the T1-weighted volumes using the pipeline described by Torrado-Carvajal et al. (23). These models contained cerebellar white and gray matter, brain white and gray matter, cerebrospinal fluid, skull, eyes, muscle, fat, and skin. We assigned a standard ${ }^{18} \mathrm{~F}-\mathrm{FDG}$ PET activity to each tissue according to relative values (gray matter, 4.0; white matter and rest of soft tissue, 1.0 ; cerebrospinal fluid and bone, 0.0$)(24,25)$, obtaining the groundtruth PET maps. Then, these ground-truth maps were projected with the $3 \mathrm{D}$ ordered-subsets expectation maximization software (26), assuming the geometry, parameters, and sinogram format of the Biograph mMR scanner (27). The system response matrix used in this case assumed a uniform gaussian point-spread function of $4 \mathrm{~mm}$ in full width at half maximum in the whole field of view. Like Hofmann et al., we neglected scatter and random coincidences to isolate the effect of the AC maps in the final reconstructed PET images (13). The projection data generated were our attenuation-free reference data.

The 511-keV AC maps derived from the CT (gold standard) and MR (pseudo-CT) images and originated using the conversion described by Nakamoto et al. (28) were forward-projected to generate the attenuation data. The forward projection is based on the same code as that used to project the PET distribution with the 4-mm point-spread function, as the blurring effects involved in the PET emission, such as positron range and noncolinearity, also affect attenuation of the $\gamma$-rays. We then used these 2 projection datasets $\left(\operatorname{Proj}_{\mu \mathrm{MR}}\right.$ and $\left.\operatorname{Proj}_{\mu \mathrm{CT}}\right)$ to generate our 


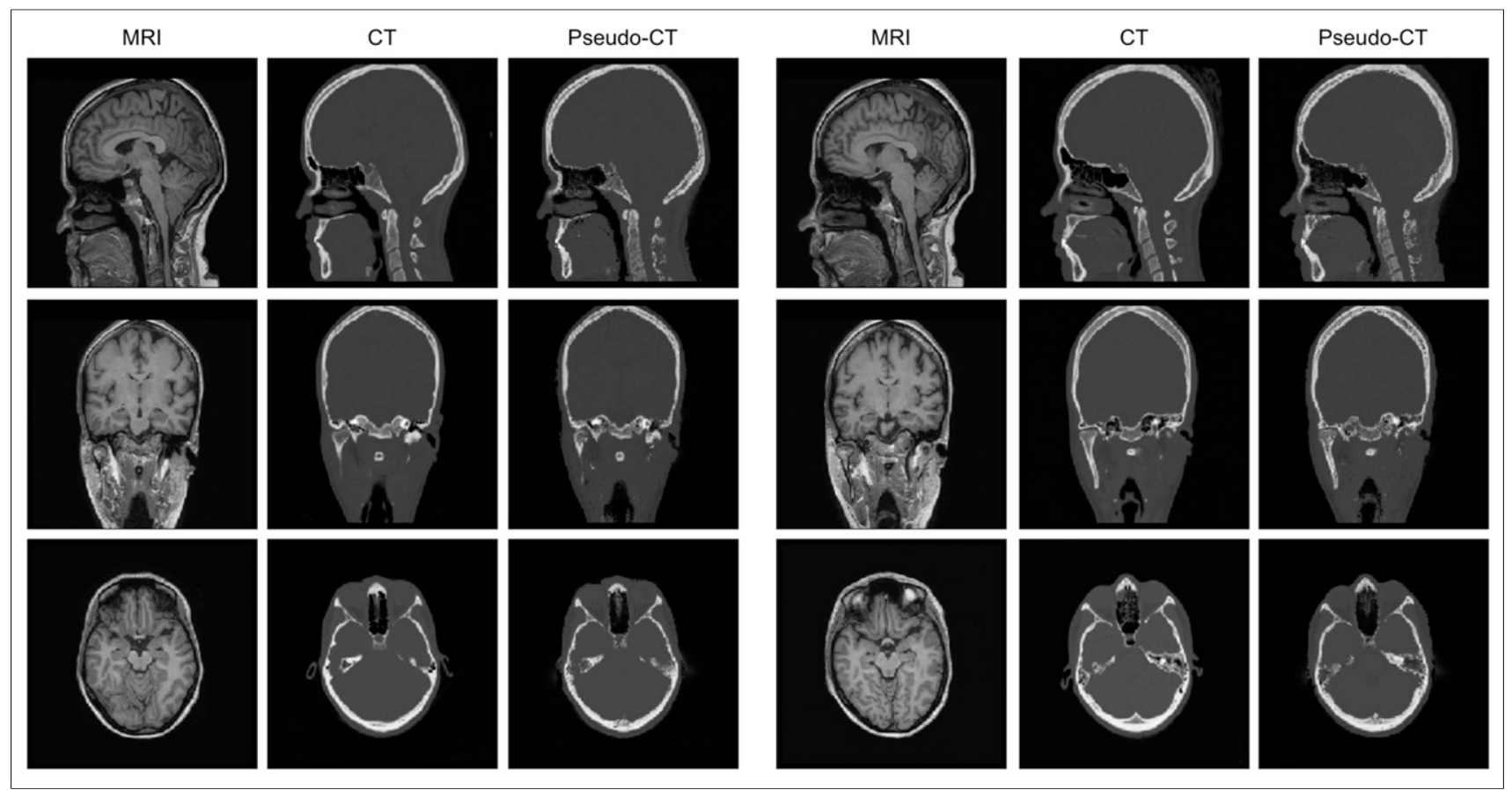

FIGURE 1. Sagittal, coronal, and axial slices of patient-specific MR (first column), patient-specific CT (second column), and synthesized pseudo-CT (third column) images of 2 subjects.

attenuation-corrected data $\left(\mathrm{PET}_{\mathrm{MR}-\mathrm{AC}}\right)$ from the attenuation-free data (PET) by following this equation:

$$
\operatorname{PET}_{\mathrm{MR}-\mathrm{AC}}=\operatorname{PET} e^{\left(\operatorname{Proj}_{\mu \mathrm{MR}}-\mathrm{Proj}_{\mu \mathrm{CT}}\right)} .
$$

\section{Image Reconstruction}

Both the attenuated projection data and the attenuation-free data were reconstructed with $3 \mathrm{D}$ ordered-subsets expectation maximization software (26) adapted to the geometry and sinogram size of the mMR scanner. We used 30 iterations and 3 subsets, and the reconstructed images consisted of $201 \times 201 \times 129$ voxels of $2 \times$ $2 \times 2 \mathrm{~mm}$ each. As indicated by Burger et al., the difference between these 2 reconstructed images depends on the reconstruction method used, but it is outside the scope of this work to analyze this effect (3).

\section{Experimental Setup}

The available data were separated into an atlas $A$ containing 10 MRI-CT pairs, $A=\left\{\left(I_{\mathrm{MR}}^{i}, I_{\mathrm{CT}}^{i}\right) i=1, \ldots, 10\right\}$, and a test set $T$ containing the 9 remaining MRI-CT pairs, $T=\left\{\left(I_{\mathrm{MR}}^{j}, I_{\mathrm{CT}}^{j}\right) j=\right.$ $1, \ldots, 9\}$.

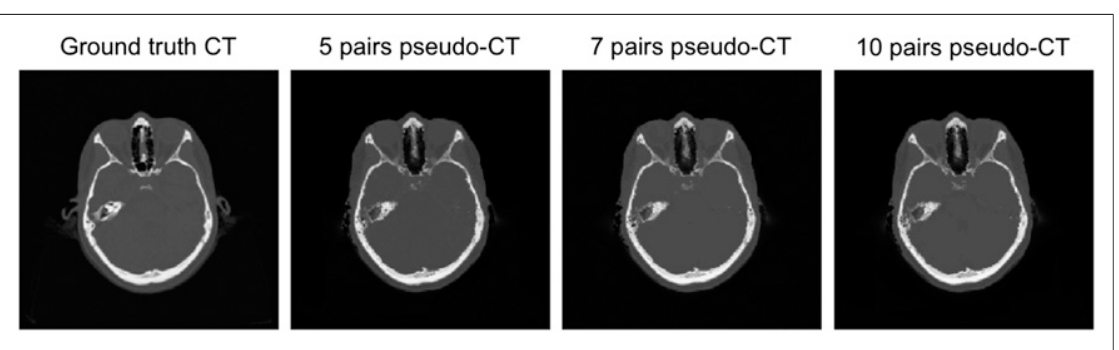

FIGURE 2. Axial patient-specific CT and pseudo-CT images synthesized using atlases of different sizes.

\section{RESULTS}

\section{Image Quality}

Figure 1 shows a complete head MRI volume, the ground-truth $\mathrm{CT}$ volume, and the synthesized pseudo-CT volume for 2 healthy subjects. An expert radiologist visually inspected the images to confirm the accuracy of our method; our pipeline worked equally well in all cases considered.

With the proposed method, the shape of the skull was estimated generally well despite patient-specific anatomic variations. The comparison between the patient-specific CT and pseudo-CT volumes showed that our method accurately estimates the ground truth, delimiting the skull contours and differentiating air from bone. Visual inspection of the results showed the high quality of the pseudo-CT estimation and the robustness of the method, which is able to capture the details of the bone spikes in nonsmooth areas such as the sinuses and the cervical vertebrae.

We also tested whether changing the size of the atlas affects the quality of the synthesized images in subjects without skull deformations, using atlases of 5, 7, and 10 datasets (Fig. 2). We used the normalized cross correlation (NCC) to quantitatively measure the quality of the synthesized pseudo-CT volumes $\left(\mathrm{I}_{2}\right)$ compared with the ground-truth CT volume $\left(\mathrm{I}_{1}\right)$, following Equation 5 .

$$
\mathrm{NCC}=\frac{1}{N} \sum_{x, y} \frac{\left(I_{1}(x, y)-\mu_{1}\right)\left(I_{2}(x, y)-\mu_{2}\right)}{\sigma_{1} \sigma_{2}} .
$$

The experimental NCC was $0.9281 \pm$ 0.0066 for the atlas with 5 datasets, $0.9294 \pm 0.0051$ for the atlas with 7 datasets, and $0.9324 \pm 0.0048$ for the atlas with 10 datasets. 


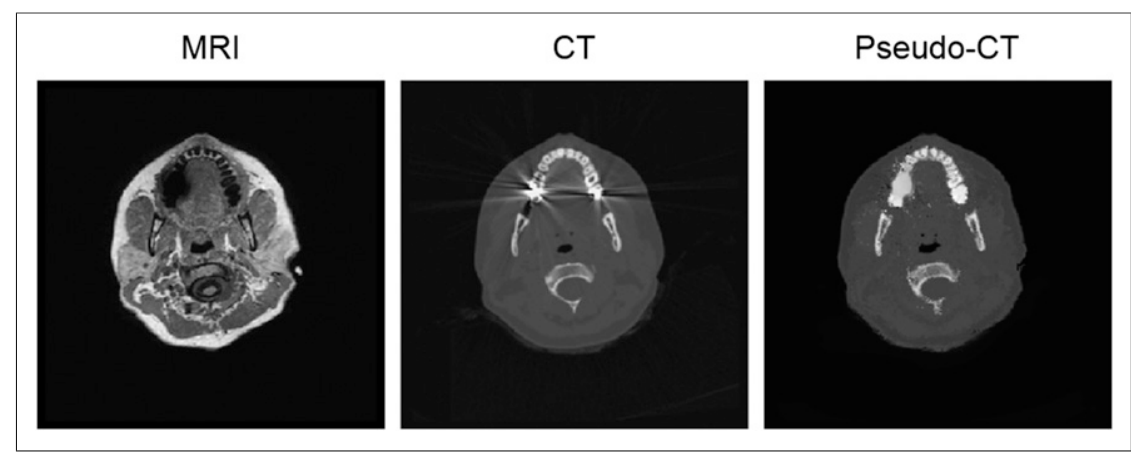

FIGURE 3. Artifacts caused by dental restorations. MR image (left) is impaired by signal loss; CT image (center) is impaired by beam hardening; pseudo-CT image (right) presents some blurring in those zones.

over the whole head, as well as the BlandAltman plot of SUVs (Fig. 5). Voxels dominated by noise (SUV $<0.01$ ) were not included in the analysis. The correlation plot showed an excellent correlation between SUVs obtained with both methods $\left(R^{2}=0.9980, P<0.0001\right)$. The Bland-Altman plot showed that the average of the differences was low $(0.0006 \pm$ 0.0594); the difference between methods tended to decrease as the average increased, accumulating the error in voxels with low SUVs.

Region-of-Interest (ROI) Analysis. ROI analysis was performed to determine differ-

Artifacts caused by dental restorations are a common problem in MRI and CT scans of the head and neck. Dental implants impair CT image quality by causing beam-hardening artifacts, MR image quality by causing signal loss, and synthesized pseudo-CT image quality by causing blurring (Fig. 3).

\section{PET Correction Quality}

Voxel-by-Voxel Analysis. Voxel-by-voxel analysis was performed to determine differences in reconstructed SUVs for all test subjects. There was an excellent match between the attenuation-free SUV images (gold standard) — attenuated and corrected with the patientspecific CT volume-and the pseudo-CT-based corrected SUV images - attenuated with the patient-specific CT volume and corrected with the synthesized pseudo-CT volume. Errors were lower than $10 \%$ of the SUVs in most voxels. Some deviations could be appreciated in subjects $\mathrm{A}$ and $\mathrm{B}$, but the error was lower than $5 \%$ on average (Fig. 4).

We also computed the voxel-by-voxel correlation between pseudo-CT-based SUV and CT-based SUV for all 9 test subjects ences in assigned attenuation coefficients and reconstructed SUVs. ROIs were defined as the original segmented tissues used to assign PET activity in the simulation.

Table 1 summarizes pseudo-CT-based and CT-based attenuation coefficients for each ROI. Paired-sample Wilcoxon signed rank tests were performed to assess whether there were differences in the mean ranks of pseudo-CT-based and CT-based attenuation coefficients. No significant difference was found in the mean CT-based attenuation coefficients, as compared with assigned pseudo-CT-based attenuation coefficients, in any ROI except the skin.

We computed the correlation between pseudo-CT-based SUV and CT-based SUV for each ROI, as well as the Bland-Altman plot of $\mathrm{SUV}_{\text {mean }}$ and $\mathrm{SUV}_{\max }$ (Fig. 6). The correlation plot for $\mathrm{SUV}_{\text {mean }}$ showed an excellent correlation between $\mathrm{SUV}_{\text {mean }}$ obtained with both methods $\left(R^{2}=0.9989, P<0.0001\right)$. The Bland-Altman plot for $\mathrm{SUV}_{\text {mean }}$ showed that the average of the differences was low $(0.0009 \pm 0.0338)$; the difference between methods did not show an increasing or decreasing trend as the

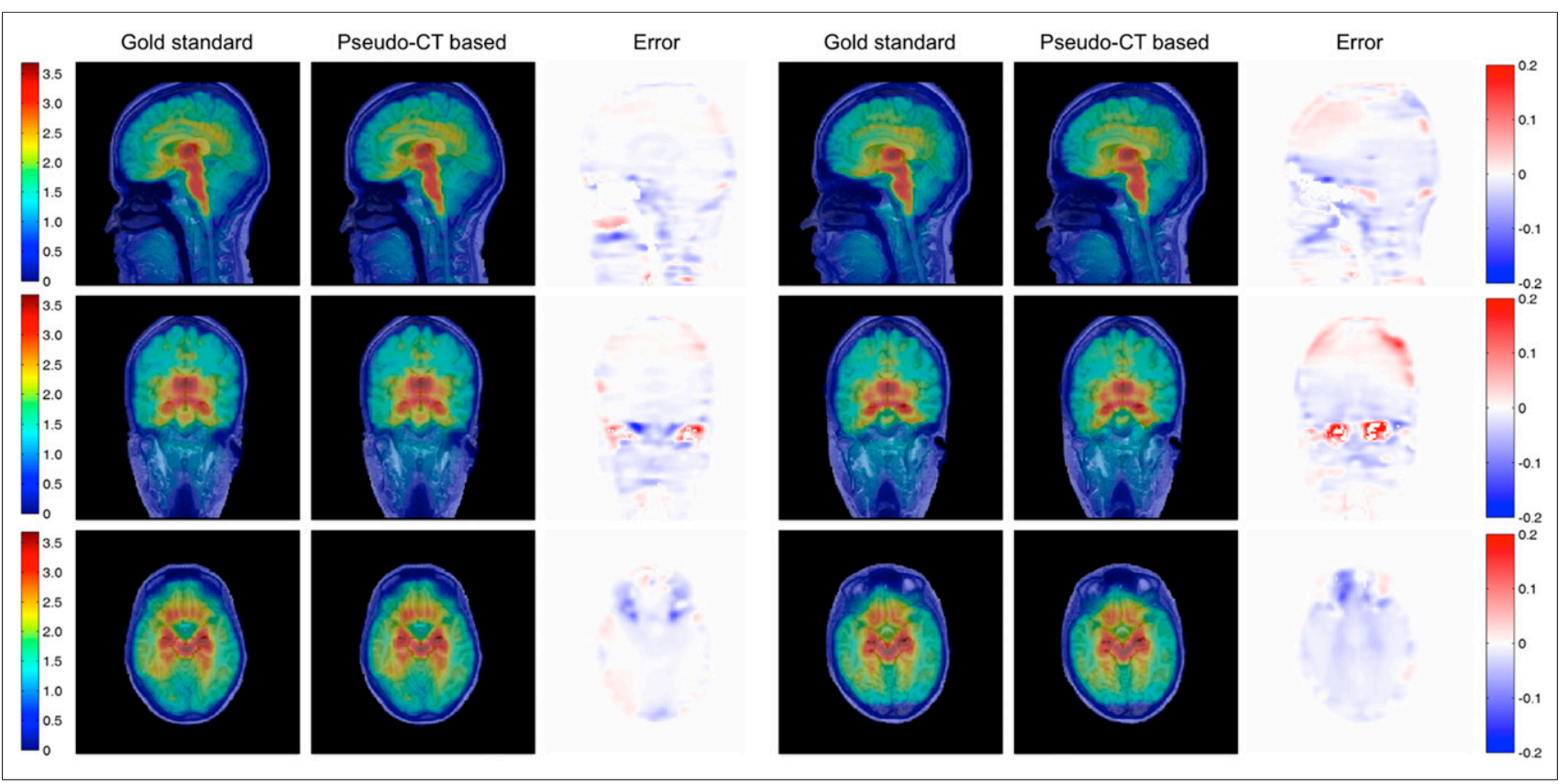

FIGURE 4. Sagittal, coronal, and axial slices of attenuation-free SUV images (first column), pseudo-CT-based corrected SUV images (second column), and error between both corrections (third column) for 2 subjects. Blue values in error maps denote decreased PET SUVs in pseudo-CTbased correction, whereas red values denote increased PET SUVs. 


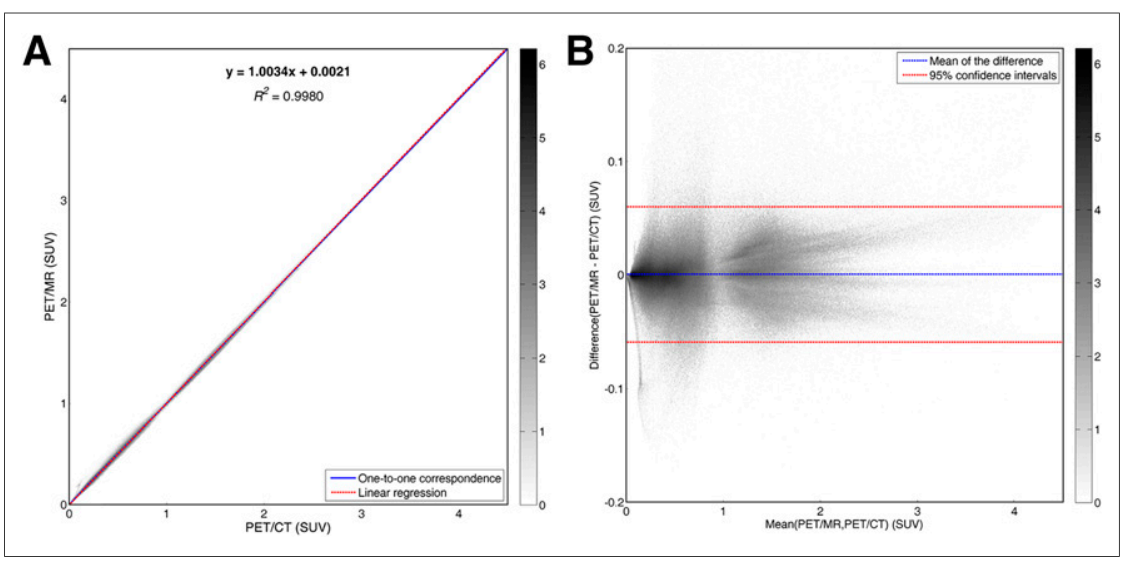

FIGURE 5. (A) Voxel-by-voxel correlation between pseudo-CT- and CT-based SUV from all subjects. (B) Bland-Altman plot of SUVs. Color bar shows density of voxels on histogram grid. second TE of $2.46 \mathrm{~ms}, 1$ excitation, acquisition matrix of $192 \times 192 \times 192$, resolution of $1.56 \times 1.56 \times 1.56 \mathrm{~mm}$, and flip angle of $10^{\circ}$, as well as a Dixon sequence with a repetition time of $3.6 \mathrm{~ms}$, first TE of $1.23 \mathrm{~ms}$, second TE of $2.46 \mathrm{~ms}$, acquisition matrix of $192 \times 126 \times 128$, resolution of $2.08 \times 2.08 \times 2.34 \mathrm{~mm}$, and flip angle of $10^{\circ}$.

Low-dose CT images were acquired on an Aquilion Prime CT scanner (Toshiba) with a matrix of $512 \times 512$, resolution of $0.43 \times 0.43 \mathrm{~mm}$, slice thickness of $1 \mathrm{~mm}$, pitch of $0.62 \mathrm{~mm}$, acquisition angle of $0^{\circ}$, voltage of $120 \mathrm{kV}$, and radiation intensity of $200 \mathrm{~mA}$.

The subject was administered 129.1 $\mathrm{MBq}$ of ${ }^{18} \mathrm{~F}$-FDG. PET data were acquired average changed. The correlation plot for $\mathrm{SUV}_{\max }$ showed a slight overestimation in the $\mathrm{SUV}_{\text {max }}$ obtained with our method $\left(R^{2}=\right.$ $0.9904, P<0.0001)$. The Bland-Altman plot for $\mathrm{SUV}_{\max }$ showed that the average of the differences was low $(0.0312 \pm 0.2345)$; the difference between methods confirmed a slight overestimation of our method in regions with low SUVs.

\section{Experiments on Clinical Data}

To perform a validation on real data, we acquired MR, PET, and CT volumes of a subject. The institutional review board (Fundación HM Hospitales) approved this study, and the subject gave written informed consent.

MR and PET images of the head were acquired on a Biograph mMR scanner (Siemens) using the body coil for excitation and an 8-channel quadrature brain coil for reception. We acquired a 3D T1weighted magnetization-prepared rapid-acquisition gradient-echo volume with a repetition time of $1,800 \mathrm{~ms}$, TE of $2.65 \mathrm{~ms}$, inversion time of $900 \mathrm{~ms}, 1$ excitation, acquisition matrix of $250 \times 250 \times$ 250 , resolution of $1 \times 1 \times 1 \mathrm{~mm}$, and flip angle of $9^{\circ}$. Furthermore, to obtain the AC map, we acquired a double-ultrashort-TE sequence with a repetition time of $11.94 \mathrm{~ms}$, first TE of $0.07 \mathrm{~ms}$,
$20 \mathrm{~min}$ after the injection at a single bed position for $15 \mathrm{~min}$. The images without AC were reconstructed using the iterative algorithm provided by the manufacturer (ordinary Poisson orderedsubsets expectation maximization) with 6 iterations and 21 subsets, a reconstruction matrix of $344 \times 344 \times 127$, and a resolution of $2.09 \times 2.09 \times 2.03 \mathrm{~mm}$. The reconstruction included a point-spread function model.

We synthesized the pseudo-CT volume using the atlas with 10 volume pairs and the same processing pipeline as in previous experiments. The reconstructed image without AC was projected with the same code as that used in the previous section. The resulting sinograms were then corrected for attenuation using the 3 different AC maps (CT-based, ultrashort-TE MRI-based, and pseudo-CT-based). The corrected sinograms were reconstructed with the $3 \mathrm{D}$ ordered-subsets expectation maximization algorithm with 30 iterations and 1 subset, a reconstruction matrix of $127 \times 127 \times 127$, and resolution of $2 \times 2 \times 2 \mathrm{~mm}$.

Figure 7 shows the MR, CT, ultrashort-TE MR, and pseudo-CT images and the result of correcting the PET data with the different volumes. The NCC was 0.5808 between the CT and the ultrashort-TE MR images and 0.8919 between the CT and pseudo-CT images.

TABLE 1

Attenuation Coefficients Based on Pseudo-CT and CT for Each ROI

\begin{tabular}{|c|c|c|c|c|}
\hline \multirow[b]{2}{*}{$\mathrm{ROI}$} & \multicolumn{2}{|c|}{ Attenuation coefficient } & \multirow[b]{2}{*}{ Absolute difference } & \multirow[b]{2}{*}{$P$} \\
\hline & Pseudo-CT-based & CT-based & & \\
\hline Cerebellar white matter & $0.094554 \pm 0.000324$ & $0.094557 \pm 0.000556$ & $0.000004 \pm 0.000570$ & 0.7969 \\
\hline Cerebellar gray matter & $0.094910 \pm 0.002787$ & $0.094919 \pm 0.003018$ & $0.000009 \pm 0.002358$ & 0.6289 \\
\hline Brain white matter & $0.094501 \pm 0.000244$ & $0.094468 \pm 0.000512$ & $0.000033 \pm 0.000530$ & 0.0938 \\
\hline Brain gray matter & $0.094982 \pm 0.004226$ & $0.094942 \pm 0.004577$ & $0.000040 \pm 0.003724$ & 0.1211 \\
\hline Cerebrospinal fluid & $0.094201 \pm 0.006186$ & $0.093993 \pm 0.002647$ & $0.000208 \pm 0.005549$ & 0.1406 \\
\hline Bone & $0.171169 \pm 0.039059$ & $0.174073 \pm 0.034212$ & $0.002904 \pm 0.028846$ & 0.2383 \\
\hline Fat & $0.096634 \pm 0.013912$ & $0.096558 \pm 0.004161$ & $0.000076 \pm 0.012901$ & 0.7969 \\
\hline Muscle & $0.087851 \pm 0.016329$ & $0.087132 \pm 0.004444$ & $0.000719 \pm 0.015747$ & 0.1563 \\
\hline Skin & $0.081767 \pm 0.022670$ & $0.087893 \pm 0.009935$ & $0.006126 \pm 0.021313$ & 0.0039 \\
\hline
\end{tabular}

Data are mean \pm SD pseudo-CT-based attenuation coefficient, CT-based attenuation coefficient, and absolute difference as obtained from Wilcoxon signed-rank test. 


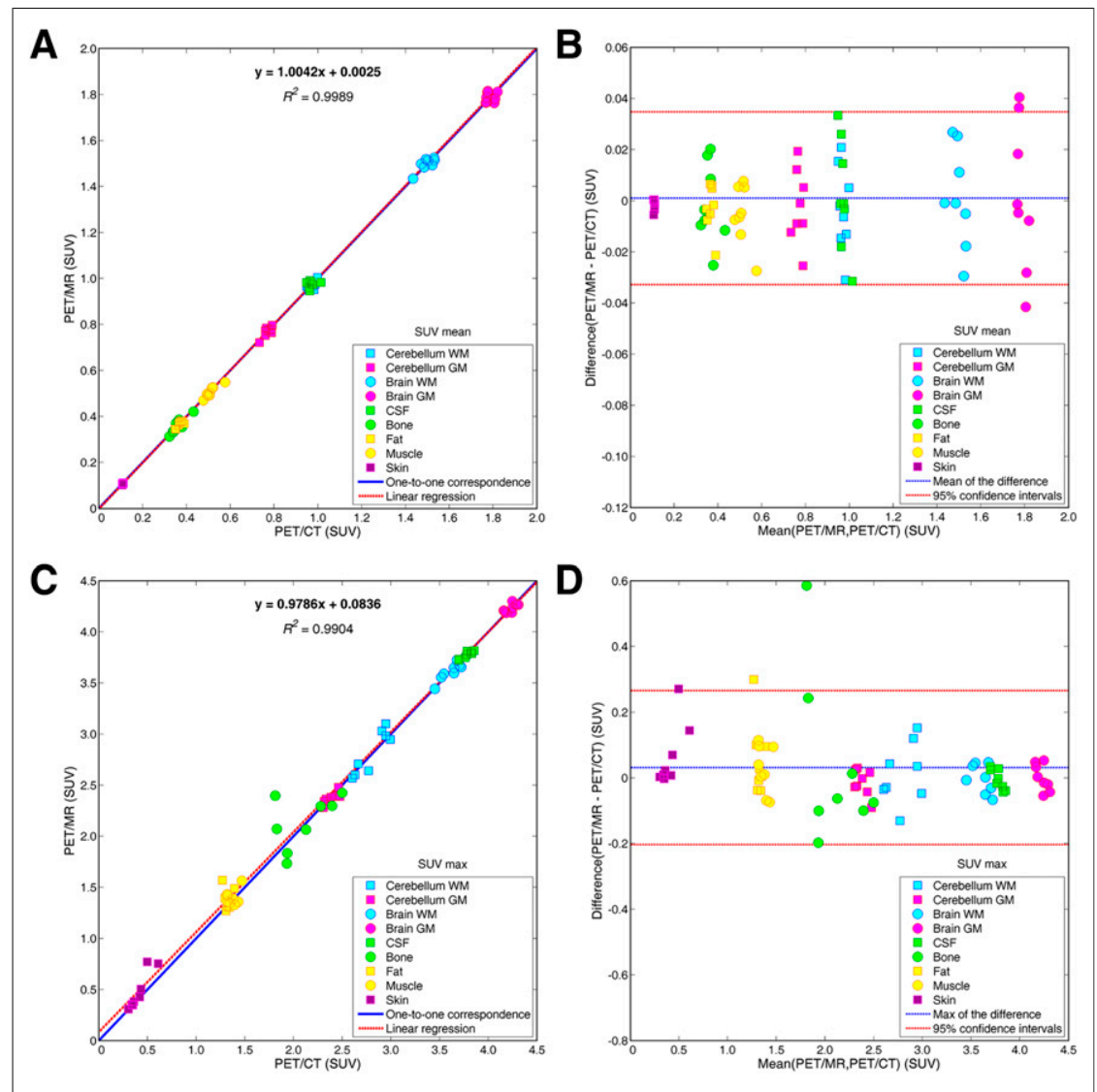

FIGURE 6. (A and C) ROI correlation between mean pseudo-CT- and CT-based SUVs (A) and between maximum pseudo-CT- and CT-based SUVs (C). (B and D) Bland-Altman plots of $\mathrm{SUV}_{\text {mean }}(\mathrm{B})$ and $\mathrm{SUV}_{\max }(\mathrm{D}) . \mathrm{CSF}=$ cerebrospinal fluid; $\mathrm{GM}=$ gray matter; $\mathrm{WM}=$ white matter.

\section{Computational Time}

For a $271 \times 271 \times 221$ volume, the complete time to synthesize the pseudo-CT volume for different numbers of images in the multiatlas case is shown in Table 2. In the worst-case scenario (atlas of 10 volumes), computation of the pseudo-CT volume in GPU took less than 9 min, 4,374 times less than with a Matlab the images.

(The MathWorks) implementation, 27 times less than with a $\mathrm{C}$ implementation, and 11 times less than with an OpenMP multithreaded $\mathrm{C}$ version using 32 threads.

All experiments were performed on a 3.1-Hz Xeon-E5 2687W (Intel), with 128 GB of random-access memory and Windows (Microsoft) Server 2008 R2 powered with a Tesla K20X GPU (Nvidia Corp.).

\section{DISCUSSION}

The generation of accurate AC maps is a basic step for PET/MRI quantification. However, ignoring bone is known to cause a distorted and biased distribution of the reconstructed SUV. Several approaches have been proposed, based mainly on a combination of MR sequences or using image registration to take advantage of a priori acquired CT data. Methods requiring the acquisition of additional MR data show a significant underestimation of activity concentration. Approaches based on nonrigid image registration are computationally intensive and are normally unable to deal with local changes in anatomy due to disease or implants.

We propose the use of a patch-based method for synthesizing a pseudo-CT volume from a single T1-weighted MR volume. Compared with nonrigid registration methods, the patch-based approach uses information from only those volumes in the atlas that actually match the patch, preventing errors due to misregistration and allowing accommodation for local morphologic changes in

Our results are in line with those recently described by Burgos et al. (15). However, our method eliminates the need for image registration, providing results with the same accuracy while allowing use of a much faster algorithm.

We have evaluated our method both qualitatively and quantitatively for PET/

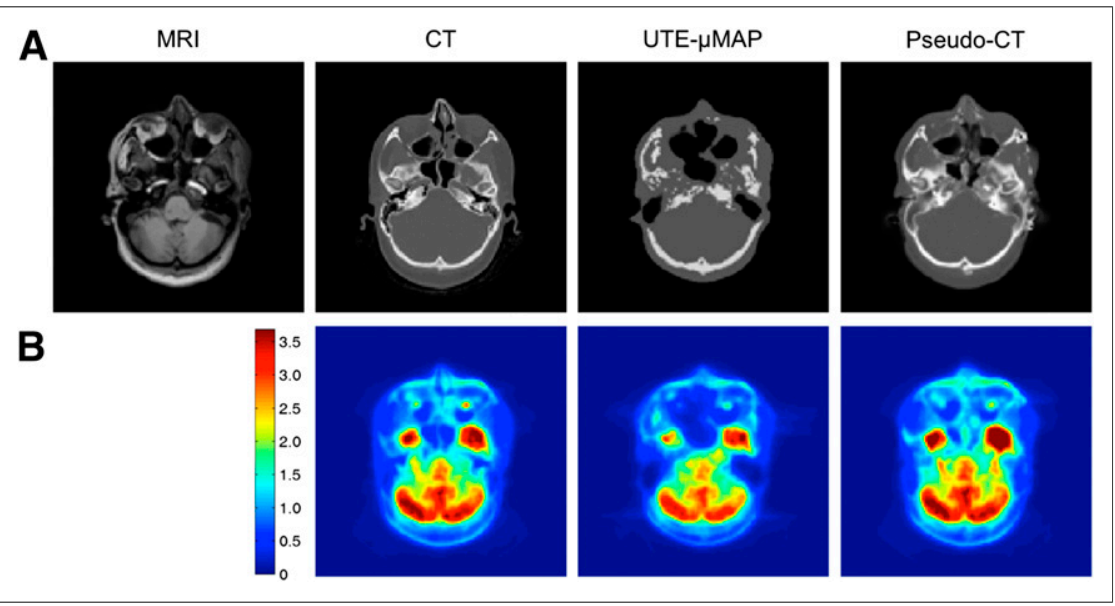

FIGURE 7. (A) Axial MR, CT, ultrashort-TE MR, and pseudo-CT images from clinical data. (B) Corrected SUV images using different AC maps. UTE- MMAP = ultrashort-TE AC map. MR correction. The visual (Fig. 1) and quantitative (Fig. 4) analyses of image quality showed that $\mathrm{CT}$ and pseudo-CT images are very similar. The high NCC values indicate that our method can accurately approximate the patient-specific $\mathrm{CT}$ volume. We also showed that in a subject with a dental restoration, results were better than with CT-based AC maps (Fig. 3). No significant differences were found in any ROI except the skin, for which the deviation was $-0.006126 \pm 0.004088$ $(P=0.0039)$ (Table 1$)$; this result may be due to the fact that the skin is the thinnest ROI and that small errors in estimation of the region can therefore have a large effect on the result. Because the CT scan is not for diagnostic purposes, the level of detail 
TABLE 2

Time to Compute Pseudo-CT Volume Using Different Implementations and Atlas Sizes

\begin{tabular}{lcccc}
\hline & \multicolumn{4}{c}{ Computational time } \\
\cline { 2 - 4 } Volumes in atlas & Matlab $(\mathrm{d})$ & $\mathrm{C}(\mathrm{min})$ & C OpenMP (min) & GPU (min) \\
\hline 5 & 11.44 & $119.91 \pm 0.02$ & $61.76 \pm 0.09$ & $4.43 \pm 0.00$ \\
7 & 16.18 & $168.08 \pm 0.04$ & $74.34 \pm 0.29$ & $6.20 \pm 0.00$ \\
10 & 26.89 & $240.11 \pm 0.14$ & & \\
& & & & \\
\hline Data are mean \pm SD (Matlab code was tested only once because of computational cost). & & \\
\hline
\end{tabular}

of the pseudo-CT scan does not need to match an actual acquired CT scan.

The attenuation coefficients that we derived in both cases from the Hounsfield values using the transformation proposed by Nakamoto et al. (28) differed slightly from the values assigned to these tissues in other publications. However, our results are independent of the transformation used, as we were comparing the pseudo-CT volume generated by our method to a gold standard CT volume and the same transformation was used in both to obtain the attenuation values.

Analysis of the absolute error maps (error = pseudo-CT-based CT-based) confirmed that there are small differences between the two approaches, mainly in the air interface zones, where the PET activity is low and usually not relevant for clinical applications (Fig. 4). The ROI analysis showed a strong correlation between attenuationfree SUV images and pseudo-CT-based corrected SUV images (Fig. 6). In evaluating the impact of the method on a normal PET brain scan, we considered only a standard ${ }^{18} \mathrm{~F}$-FDG biodistribution but expect similar small deviations with other radiotracers.

We have also tested our method on real clinical data. Using the real CT volume of the subject as a gold standard, we compared the pseudo-CT image obtained with our method and the ultrashort-TE MR image currently used for AC in the mMR scanner. The ultrashort-TE MR image is not able to differentiate air from bone in certain regions, leading to errors in the final PET image (Fig. 7). The NCC for the clinical data is lower than that for the test data, possibly because of the different scanners and sequences used to acquire the clinical data and the atlas volumes. As future research, we will design similarity measures that are robust to such changes.

Our study neglected the impact of possible deviations in the MR-derived AC map when scatter correction was considered. The distribution of scatter in the sinogram is usually smooth, and the effects of AC map deviations on scatter correction are averaged over many sinogram bins. Therefore, small deviations in the AC map will have a modest impact on scatter correction, especially as the scaling factor used to fit the estimated scatter distribution to the experimental data would remove any overall bias that might be introduced. Our study also neglected the effect of possible errors in modeling the attenuation of the MR coils (12). However, this effect can be controlled in clinical practice with appropriate scanner modeling, calibrations, and acquisition protocols.

The method is highly parallelizable, as each voxel is computed independently. This has allowed implementation of a GPU version that provides a complete pseudo-CT volume during a time similar to that needed to acquire an actual CT scan. Because there is still room for improvement in the GPU implementation, it may be possible to speed up the algorithm further.
We have validated our approach using a set of 10 whole-head volume pairs from healthy subjects, in contrast to the typical brain studies that do not include the neck, which is difficult to synthesize because of its anatomic complexity and variability.

Our technique could, in theory, be applied to other regions of the body. Registration-based approaches are not so straightforward as our approach in other regions, because of stronger intersubject anatomic differences. In a real clinical application, the atlas should be designed to include the necessary anatomic heterogeneity. The design of specific databases that take into account skull lesions will be a future line of research. However, our study suggests that SUVs measured on PET/MR and those measured on PET/CT are close enough; this finding makes our approach feasible for qualitative interpretation of PET scans for diagnostic purposes. The method should be further validated on images with distinct regions, such as tumors.

\section{CONCLUSION}

In this work, we showed how the use of patch-based techniques to estimate pseudo-CT images from T1-weighted MR images allows determination of accurate AC maps for use in hybrid PET/MR systems. The proposed method estimates a pseudo-CT volume with similar accuracy to a patient-specific CT volume, without the need for a registration step. Atlas-based approaches avoid the oversimplification of most of the previously proposed segmented MR image-based methods, which assume that all voxels in the same tissue type should have the same attenuation coefficients. The GPU implementation led to a substantial decrease in computational time, making the approach suitable for real applications.

\section{DISCLOSURE}

The costs of publication of this article were defrayed in part by the payment of page charges. Therefore, and solely to indicate this fact, this article is hereby marked "advertisement" in accordance with 18 USC section 1734. This project was supported in part by Consejería de Educación, Juventud y Deporte de la Comunidad de Madrid, through the Madrid-MIT M+Visión Consortium and the Seventh Framework Programme of the European Union, and project TEC2012-39095-C03-01 of the Spanish Ministry of Economy and Competitiveness. No other potential conflict of interest relevant to this article was reported.

\section{ACKNOWLEDGMENT}

We thank the anonymous reviewers for their valuable comments and suggestions that helped to improve the quality of the study. 


\section{REFERENCES}

1. Shao Y, Cherry SR, Farahani K, et al. Simultaneous PET and MR imaging. Phys Med Biol. 1997;42:1965-1970.

2. Wagenknecht G, Kaiser HJ, Mottaghy FM, Herzog H. MRI for attenuation correction in PET: methods and challenges. MAGMA. 2013;26:99-113.

3. Burger C, Goerres G, Schoenes S, Buck A, Lonn AH, Von Schulthess GK. PET attenuation coefficients from CT images: experimental evaluation of the transformation of CT into PET 511-keV attenuation coefficients. Eur J Nucl Med Mol Imaging. 2002;29:922-927.

4. Andersen FL, Ladefoged CN, Beyer T, et al. Combined PET/MR imaging in neurology: MR-based attenuation correction implies a strong spatial bias when ignoring bone. Neuroimage. 2014;84:206-216.

5. Hofmann M, Pichler B, Schölkopf B, Beyer T. Towards quantitative PET/MRI: a review of MR-based attenuation correction techniques. Eur J Nucl Med Mol Imaging. 2009;36(suppl):S93-S104.

6. Dogdas B, Shattuck DW, Leahy RM. Segmentation of skull and scalp in 3-D human MRI using mathematical morphology. Hum Brain Mapp. 2005;26:273-285.

7. Wang D, Shi L, Chu WC, Cheng JC, Heng PA. Segmentation of human skull in MRI using statistical shape information from CT data. J Magn Reson Imaging. 2009;30:490-498.

8. Martinez-Möller A, Souvatzoglou M, Delso G, et al. Tissue classification as a potential approach for attenuation correction in whole-body PET/MRI: evaluation with PET/CT data. J Nucl Med. 2009;50:520-526.

9. Robson MD, Bydder GM. Clinical ultrashort echo time imaging of bone and other connective tissues. NMR Biomed. 2006;19:765-780.

10. Keereman V, Fierens Y, Broux T, De Deene Y, Lonneux M, Vandenberghe S. MRI-based attenuation correction for PET/MRI using ultrashort echo time sequences. J Nucl Med. 2010;51:812-818.

11. Torrado-Carvajal A, Herraiz JL, Hernandez-Tamames JA, et al. Multi-atlas and label fusion approach for patient-specific MRI based skull estimation. Magn Reson Med. May 18, 2015 [Epub ahead of print].

12. Visvikis D, Monnier F, Bert J, Hatt M, Fayad H. PET/MR attenuation correction: where have we come from and where are we going? Eur J Nucl Med Mol Imaging. 2014;41:1172-1175.

13. Hofmann M, Steinke F, Scheel V, et al. MRI-based attenuation correction for PET/MRI: a novel approach combining pattern recognition and atlas registration. J Nucl Med. 2008;49:1875-1883.

14. Izquierdo-Garcia D, Hansen AE, Förster S, et al. An SPM8-based approach for attenuation correction combining segmentation and nonrigid template formation: application to simultaneous PET/MR brain imaging. J Nucl Med. 2014;55:1825-1830.
15. Burgos N, Cardoso M J, Thielemans K, et al. Attenuation correction synthesis for hybrid PET-MR scanners: application to brain studies. IEEE Trans Med Imaging. 2014 33: 2332-41.

16. Dickson JC, O'Meara C, Barnes A. A comparison of CT- and MR-based attenuation correction in neurological PET. Eur J Nucl Med Mol Imaging. 2014;41: 1176-1189.

17. Rousseau F, Habas PA, Studholme C. A supervised patch-based approach for human brain labeling. IEEE Trans Med Imaging. 2011;30:1852-1862.

18. Buades A, Coll B, Morel JM. A review of image denoising algorithms, with a new one. Multiscale Model Simul. 2005;4:490-530.

19. Coupé P, Manjón JV, Fonov V, Pruessner J, Robles M, Collins DL. Patch-based segmentation using expert priors: application to hippocampus and ventricle segmentation. Neuroimage. 2011;54:940-954.

20. Bai W, Shi W, O'Regan DP, et al. A probabilistic patch-based label fusion model for multi-atlas segmentation with registration refinement: application to cardiac MR images. IEEE Trans Med Imaging. 2013;32:1302-1315.

21. Ye DH, Zikic D, Glocker B, Criminisi A, Konukoglu E. Modality propagation: coherent synthesis of subject-specific scans with data-driven regularization. Med Image Comput Comput Assist Interv. 2013;16:606-613.

22. Kikinis R, Pieper SD, Vosburgh KG. 3D slicer: a platform for subject-specific image analysis, visualization, and clinical support. In: Jolesz FA, ed. Intraoperative Imaging and Image-Guided Therapy. New York, NY: Springer; 2014: 277-289.

23. Torrado-Carvajal A, Hernandez-Tamames JA, Herraiz JL, et al. Automatic segmentation pipeline for patient-specific MRI tissue models. Presented at: Proceedings of the Joint Annual Meeting of the ISMRM-ESMRMB; Milan, Italy; May 1-16, 2014.

24. Vunckx K, Dupont P, Goffin K, Van Paesschen W, Van Laere K, Nuyts J. Voxelbased comparison of state-of-the-art reconstruction algorithms for ${ }^{18} \mathrm{~F}-\mathrm{FDG}$ PET brain imaging using simulated and clinical data. Neuroimage. 2014;102:875-884.

25. Catana C, van der Kouwe A, Benner T, et al. Towards implementing an MRbased PET attenuation correction method for neurological studies on the MRPET brain prototype. J Nucl Med. 2010;51:1431-1438.

26. Herraiz JL, España S, Cabido R, et al. GPU-based fast iterative reconstruction of fully 3-D PET sinograms. IEEE Trans Nucl Sci. 2011;58:2257-2263.

27. Delso G, Fürst S, Jakoby B, et al. Performance measurements of the Siemens mMR integrated whole-body PET/MR scanner. J Nucl Med. 2011;52:1914-1922.

28. Nakamoto Y, Osman M, Cohade C, et al. PET/CT: comparison of quantitative tracer uptake between germanium and $\mathrm{CT}$ transmission attenuation-corrected images. J Nucl Med. 2002;43:1137-1143. 